\title{
Improved Photometric Calibrations for Red Stars Observed with the SDSS Photometric Telescope
}

James R. A. Davenport

Western Washington University, james.davenport@wwu.edu

John J. Bochanski

Kevin R. Covey

WesternWashingtonUniversity, kevin.covey@wwu.edu

Suzanne L. Hawley

Andrew A. West

See next page for additional authors

Follow this and additional works at: https://cedar.wwu.edu/physicsastronomy_facpubs

Part of the Stars, Interstellar Medium and the Galaxy Commons

\section{Recommended Citation}

Davenport, James R. A.; Bochanski, John J.; Covey, Kevin R.; Hawley, Suzanne L.; West, Andrew A.; and Schneider, Doanld P., "Improved Photometric Calibrations for Red Stars Observed with the SDSS Photometric Telescope" (2007). Physics \& Astronomy. 22. https://cedar.wwu.edu/physicsastronomy_facpubs/22 
Authors

James R. A. Davenport, John J. Bochanski, Kevin R. Covey, Suzanne L. Hawley, Andrew A. West, and Doanld P. Schneider 


\title{
IMPROVED PHOTOMETRIC CALIBRATIONS FOR RED STARS OBSERVED WITH THE SDSS PHOTOMETRIC TELESCOPE
}

\author{
James R. A. Davenport, ${ }^{1,2}$ John J. Bochanski, ${ }^{2}$ Kevin R. Covey, ${ }^{3}$ Suzanne L. Hawley, ${ }^{2}$ \\ Andrew A. West, ${ }^{4}$ and Donald P. Schneider ${ }^{5}$ \\ Received 2007 July 12; accepted 2007 July 26
}

\begin{abstract}
We present a new set of photometric transformations for red stars observed with the Sloan Digital Sky Survey (SDSS) $0.5 \mathrm{~m}$ Photometric Telescope (PT) and the SDSS $2.5 \mathrm{~m}$ telescope at the Apache Point Observatory in New Mexico. Nightly PT observations of US Naval Observatory standards are used to determine extinction corrections and calibration terms for SDSS $2.5 \mathrm{~m}$ photometry. Systematic differences between the PT and native SDSS $2.5 \mathrm{~m}$ ugriz photometry require conversions between the two systems which have previously been undefined for the reddest stars. By matching 43,000 stars observed with both the PT and SDSS $2.5 \mathrm{~m}$, we extend the present relations to include low-mass stars with colors $0.6 \leq r-i \leq 1.7$. These corrections will allow us to place photometry of bright, low-mass trigonometric parallax stars previously observed with the PT on the $2.5 \mathrm{~m}$ system. We present new transformation equations and discuss applications of these data to future low-mass star studies using the SDSS.
\end{abstract}

Key words: stars: late-type — stars: low-mass, brown dwarfs — surveys

\section{INTRODUCTION}

The study of low-mass stars has blossomed with the advent of large-scale surveys. Due to their low intrinsic luminosity $\left(\lesssim 10^{-2} L_{\odot}\right)$ and the small fraction of light emitted in the optical band, most prior large-area surveys have been limited to nearby objects. Recent surveys such as the Sloan Digital Sky Survey (SDSS; York et al. 2000) and the Two Micron All Sky Survey (2MASS; Skrutskie et al. 1997) have now produced extensive volumes of photometry and spectroscopy on cool stars (e.g., Strauss et al. 1999; Hawley et al. 2002; Walkowicz et al. 2004; West et al. 2004, 2005, 2006; Bochanski et al. 2007a; Covey et al. 2007). Studying these faint neighbors gives us insight into the most numerous stellar population in the Galaxy, probing both young and old subsets of low-mass stars, and placing their properties, such as abundances and dynamics, in a Galactic context (Hawley et al. 2002; Chiu et al. 2006; S. A. Metchev et al. 2008, in preparation). Ongoing studies seek to measure the luminosity function and mass function of low-mass stars (Covey et al. 2007) and the dynamics of the thin and thick disks of the Milky Way (Bochanski et al. 2007b), and to characterize magnetic activity and dynamical heating in the Galactic disk (West et al. 2004, 2006, 2007).

These studies are enabled by SDSS photometry due to its high sensitivity, near-infrared bandpasses, and accurate photometry, which achieves a relative precision of $2 \%-3 \%$ (Ivezić et al. 2004). However, creating a suitable calibration onto an absolute system for SDSS photometry over the entire range of stellar colors poses a great challenge. US Naval Observatory (USNO) measurements of 158 standard stars form the foundation for the SDSS photometric calibration (Fukugita et al. 1996; Smith et al. 2002). These standards are observed on the $u^{\prime} g^{\prime} r^{\prime} i^{\prime} z^{\prime}$ system and mainly comprise early-type $(<$ spectral class $\mathrm{M})$ stars too bright

\footnotetext{
${ }^{1}$ Corresponding author: uwjim@astro.washington.edu.

2 Department of Astronomy, University of Washington, Box 351580, Seattle, WA 98195, USA.

${ }^{3}$ Harvard-Smithsonian Center for Astrophysics, 60 Garden Street, MS-72, Cambridge, MA 02138, USA.

4 Astronomy Department, University of California, 601 Campbell Hall, Berkeley, CA 94720-3411, USA.

5 Department of Astronomy and Astrophysics, The Pennsylvania State University, 525 Davey Lab, University Park, PA 16802, USA.
}

to be imaged with the SDSS $2.5 \mathrm{~m}$, but which have well-defined magnitudes.

The SDSS Photometric Telescope (PT), with an aperture of $0.5 \mathrm{~m}$, is employed to transfer the photometric calibration from the USNO measurements to the SDSS. Located alongside the SDSS $2.5 \mathrm{~m}$ telescope (Gunn et al. 2006) at Apache Point Observatory (APO), the PT provides nightly observations on the $u^{\prime} g^{\prime} r^{\prime} i^{\prime} z^{\prime}$ photometric system. ${ }^{6}$ By imaging patches of sky coincident with the SDSS nightly footprint, the PT is able to provide a robust calibration between the $u^{\prime} g^{\prime} r^{\prime} i^{\prime} z^{\prime}$ and native SDSS $2.5 \mathrm{~m}$ ugriz systems. Tucker et al. (2006, hereafter T06) details this three-telescope calibration method, including the data reduction pipeline (MTPIPE) created for the PT.

Unfortunately, the transformations described in T06 are defined only for limited ranges in color-space. These ranges, listed in equation (1), are too blue for low-mass star studies (Bochanski et al. 2007a; West et al. 2005):

$$
\begin{aligned}
& 0.70 \leq(u-g)_{\mathrm{PT}} \leq 2.70, \\
& 0.15 \leq(g-r)_{\mathrm{PT}} \leq 1.20, \\
&-0.10 \leq(r-i)_{\mathrm{PT}} \leq 0.60, \\
&-0.20 \leq(i-z)_{\mathrm{PT}} \leq 0.40 .
\end{aligned}
$$

In particular, $\left(\right.$ ugriz $_{\mathrm{PT}}$ photometry ${ }^{7}$ diverges from the $(\text { ugriz })_{\mathrm{SDSS}}$ stellar locus for stars redder than $(r-i)_{\text {SDSS }} \geq 0.6$, as shown in Figure 1. These systematic offsets are not unexpected, due to physical differences between the $u^{\prime} g^{\prime} r^{\prime} i^{\prime} z^{\prime}$ and ugriz filters and the complex calibration between the two native telescope systems (T06). Additional correction terms are needed to rectify this systematic offset, thus placing red stars observed with the PT on the native SDSS 2.5 m system.

\footnotetext{
${ }^{6}$ The PT telescope uses a $u^{\prime} g^{\prime} r^{\prime} i^{\prime} z^{\prime}$ filter set, and the PT data are first calibrated to the USNO $u^{\prime} g^{\prime} r^{\prime} i^{\prime} z^{\prime}$ system before being transformed into SDSS ugriz magnitudes.

7 The expression (ugriz) PT denotes PT photometry which has been transformed from the native PT $u^{\prime} g^{\prime} r^{\prime} i^{\prime} z^{\prime}$ to the standard SDSS $2.5 \mathrm{~m} u g r i z$ system using the T06 calibrations. We adopt the subscript notation $(\text { ugriz })_{\mathrm{SDSS}}$ for native SDSS $2.5 \mathrm{~m}$ photometry.
} 


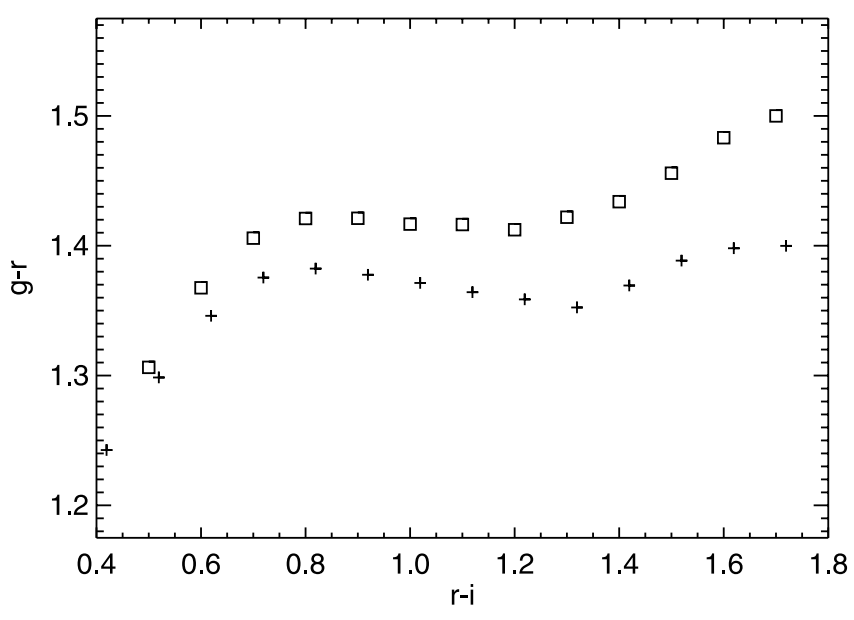

Fig. 1.- Median $(g-r),(r-i)$ colors of low-mass stars. The SDSS data (open

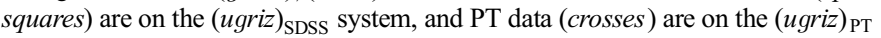
system. Note the $\sim 0.1$ mag offset between the two stellar loci for red $(r-i)$ colors.

An important application of these calibrations would permit PT observations of bright trigonometric parallax stars to be placed

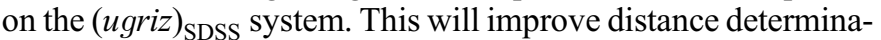
tions from photometric parallaxes for low-mass stars, which would greatly benefit many of the studies described above.

In this paper we present analysis of photometry for $\sim 43,000$ red point sources with both SDSS $2.5 \mathrm{~m}$ and PT detections. These data are used to derive transformations between the $(\text { ugriz })_{\mathrm{PT}}$ and

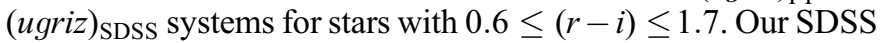
and PT sample selection is described in $\S 2$. In $\S 3$ we present our transformation equations for red stars between the two systems. A discussion of the implications of this improved calibration for low-mass star studies is presented in $\S 4$.

\section{SAMPLE SELECTION}

\subsection{SDSS Sample}

Our data are taken from the $8000 \mathrm{deg}^{2}$ of the SDSS Data Release 5 (DR5; Adelman-McCarthy et al. 2007). Technical descriptions of the survey can be found in the references in the introduction, as well as in Gunn et al. (1998), Hogg et al. (2001), Pier et al. (2003), and Stoughton et al. (2002).

To generate a photometric catalog of low-mass stars in the

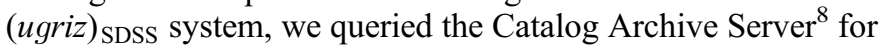
photometric observations classified as stars with the colors of $r-i>0.5$ and $i-z>0.3$ within the DR5 footprint. To ensure accurate point-spread function (PSF) photometry, several photometric quality flags were imposed on the data. ${ }^{9}$ The final sample contained approximately 13.6 million stars. The brightness distribution of these objects is shown in Figure 2. Because our goal was only to compare the photometric response of red stars between the ugriz and $u^{\prime} g^{\prime} r^{\prime} i^{\prime} z^{\prime}$ systems, no corrections for Galactic extinction were used.

\subsection{PT Sample}

During normal photometric operations on the SDSS $2.5 \mathrm{~m}$ telescope, the PT automatically images overlapping patches of the sky for calibration. These patches are roughly $15^{\circ}$ apart and cover every

\footnotetext{
${ }^{8}$ See http://cas.sdss.org/dr5/en/.

${ }^{9}$ Specifically, we required detections in BINNED1 in the $r, i$, and $z$ bands. We also selected against $r, i$, and $z$ observations with the EDGE, NOPROFILE, PEAKCENTER, NOTCHECKED, PSF FLUX INTERP, SATURATED, DEBLEND_NOPEAK, INTERP_CENTER, COSMIC_RAY, or BAD_COUNTS_ERROR flags set, as well as observations with PSF magnitude errors greater than $0.2 \mathrm{mag}$.
}

stripe (great circle path of scan) in the SDSS survey. Typically, these images are used to create photometric zero-point "anchors" for the SDSS data onto the USNO system. However, these patches also contain many stars which are not used in the SDSS calibration, but are reduced and saved by MTPIPE (T06). Over five million background star observations were made with the PT during the first $6 \mathrm{yr}$ of SDSS observations. These reduced MTPIPE data are available via the SDSS Data Archive Server. ${ }^{10}$

After removing stars which MTPIPE flagged as having bad photometry (magnitude $=-100$ ), the PT sample was selected using two color cuts. Low-mass candidates were selected by requiring $(r-i)_{\mathrm{PT}}>0.5$ (Bochanski et al. 2007a). A further color cut of $1.1 \leq(g-r)_{\text {PT }} \leq 1.7$ was used to isolate the low-mass stellar locus, removing carbon stars (Fan 1999). This process yielded $\sim 345,000$ stars with red colors which have accurate PT magnitudes. The PT subsample has mean magnitude errors of $\sigma\left(g_{\mathrm{PT}}\right)=$ $0.05, \sigma\left(r_{\mathrm{PT}}\right)=0.07, \sigma\left(i_{\mathrm{PT}}\right)=0.08$, and $\sigma\left(z_{\mathrm{PT}}\right)=0.06$. We did not use the $u_{\mathrm{PT}}$ data due to the lack of good photometry for these red stars; only $\sim 12,000$ stars in the PT sample have good $u_{\mathrm{PT}}$ photometry $\left[\sigma\left(u_{\mathrm{PT}}\right) \leq 0.05\right]$.

\subsection{Matched Sample}

To facilitate matching between the two samples, we imposed a bright magnitude limit on the PT sample to isolate stars potentially observed by both telescopes (see Fig. 2). In particular, the following magnitude cuts were used on the PT sample: $15.4 \leq g_{\mathrm{PT}} \leq 21.5$, $14.6 \leq r_{\mathrm{PT}} \leq 20.0,14.0 \leq i_{\mathrm{PT}} \leq 19.7$, and $14.1 \leq z_{\mathrm{PT}} \leq 19.5$.

The PT and SDSS samples were matched by celestial position, yielding $42,912 \mathrm{PT}-2.5 \mathrm{~m}$ matches within a search radius of $0.5^{\prime \prime}$. The SDSS $2.5 \mathrm{~m}$ astrometry is accurate to $0.045^{\prime \prime}$, while the PT astrometry is accurate to $\sim 1^{\prime \prime}$. As we are not trying to find a complete sample, we choose a conservative search radius $\left(0.5^{\prime \prime}\right)$ to minimize mismatches, and still obtain a large number of matches. Since typical proper motions of these stars were small over the limited time range of PT observations (2002-2007), the loss of some high proper motion stars was negligible.

\section{RESULTS}

\subsection{Determining Offsets}

Using the matched sample described in $\S 2.3$, the magnitude differences between the two systems were determined for each griz filter as functions of PT color (see Fig. 3). Least-squares polynomial fits to the data provide quantitative offsets between the $(\text { griz })_{\mathrm{PT}}$ and (griz) $)_{\text {SDSS }}$ systems (see $\S 3.2$ ). Two-part piecewise functions were used for the $g_{\mathrm{PT}}$ and $r_{\mathrm{PT}}$ corrections which were functions of $(r-i)_{\mathrm{PT}}$. These fits both had breaks at $(r-i)_{\mathrm{PT}}=1.25$ and were found to be better fits to the data than single polynomials.

The systematic selection of stars in the blue end of the data shown in Figure 3 occurs due to the color cuts chosen in the PT and SDSS sample selection. Our fitting regions, shown in Figure 3 and listed in equation (2), avoid these areas:

$$
\begin{aligned}
& 1.20 \leq(g-r)_{\mathrm{PT}} \leq 1.55 \\
& 0.60 \leq(r-i)_{\mathrm{PT}} \leq 1.70 \\
& 0.35 \leq(i-z)_{\mathrm{PT}} \leq 0.80
\end{aligned}
$$

To remove any strong outliers (i.e., visual binaries, mismatches, and flares), our fits are weighted by photometric error and are based only on data with a photometric error less than 0.05 mag in every color band for each observation.

\footnotetext{
${ }^{10}$ See http://das.sdss.org/PT/
} 

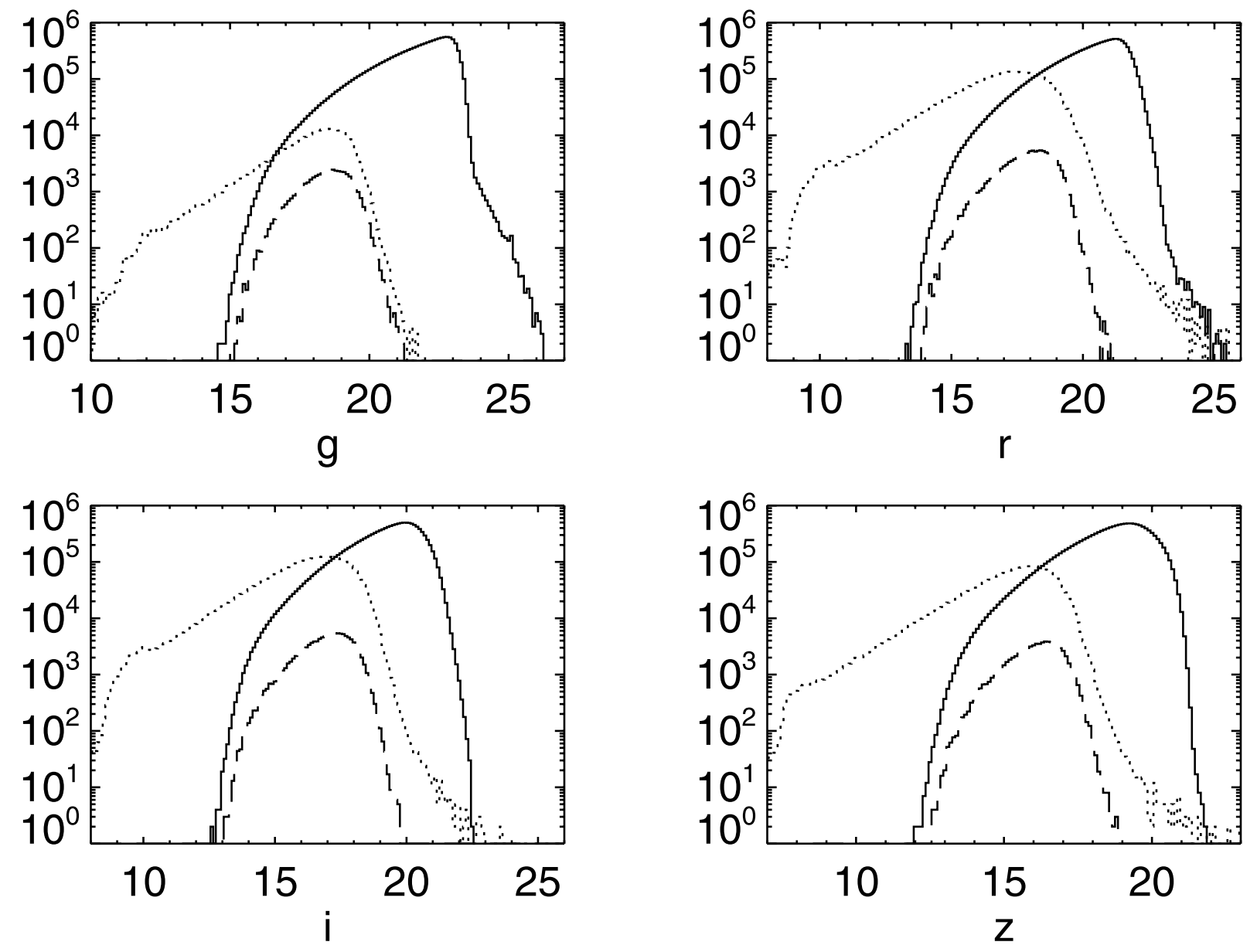

FIG. 2.- Histograms of the full five million PT (dotted line) and 13.6 million SDSS (solid line) data sets. Note the brighter limits of the smaller PT telescope. The matched subsample of $\sim 43,000$ stars is shown as the dashed line for each filter.

\subsection{Transformations}

The following equations produce the fits shown in Figure 3. These fits provide the best transformation of $(\text { griz })_{\mathrm{PT}}$ data to the native $(\text { griz })_{\text {SDSS }}$ system for red stars:

$$
\begin{aligned}
g_{\mathrm{SDSS}}\left[0.6 \leq(r-i)_{\mathrm{PT}}<1.25\right]= & g_{\mathrm{PT}}+0.142 \\
& -0.514(r-i)_{\mathrm{PT}} \\
& +0.647(r-i)_{\mathrm{PT}}^{2} \\
& -0.241(r-i)_{\mathrm{PT}}^{3} \pm 0.072, \\
g_{\mathrm{SDSS}}\left[1.25 \leq(r-i)_{\mathrm{PT}} \leq 1.7\right]= & g_{\mathrm{PT}}-2.511 \\
& +5.391(r-i)_{\mathrm{PT}} \\
& -3.787(r-i)_{\mathrm{PT}}^{2} \\
& +0.885(r-i)_{\mathrm{PT}}^{3} \pm 0.082
\end{aligned}
$$

$r_{\mathrm{SDSS}}\left[0.6 \leq(r-i)_{\mathrm{PT}}<1.25\right]=r_{\mathrm{PT}}+0.116$

$$
\begin{aligned}
& -0.339(r-i)_{\mathrm{PT}} \\
& +0.301(r-i)_{\mathrm{PT}}^{2} \\
& -0.091(r-i)_{\mathrm{PT}}^{3} \pm 0.043,
\end{aligned}
$$

$r_{\mathrm{SDSS}}\left[1.25 \leq(r-i)_{\mathrm{PT}} \leq 1.7\right]=r_{\mathrm{PT}}+0.003$

$$
-0.009(r-i)_{\mathrm{PT}} \pm 0.055
$$

$$
\begin{aligned}
i_{\mathrm{SDSS}}\left[0.6 \leq(r-i)_{\mathrm{PT}} \leq 1.7\right]= & i_{\mathrm{PT}}-0.019 \\
& +0.082(r-i)_{\mathrm{PT}} \\
& -0.068(r-i)_{\mathrm{PT}}^{2} \\
& +0.024(r-i)_{\mathrm{PT}}^{3} \pm 0.033 ; \\
z_{\mathrm{SDSS}}[0.35 \leq(i-z) \leq 0.8]= & z_{\mathrm{PT}}-0.285+1.566(i-z)_{\mathrm{PT}} \\
& -2.637(i-z)_{\mathrm{PT}}^{2} \\
& +1.488(i-z)_{\mathrm{PT}}^{3} \pm 0.050 .
\end{aligned}
$$

These equations are valid for the color ranges listed in equation (2), and should only be employed within these ranges. Also, these corrections are applicable only to PT observations which have been reduced by MTPIPE and not USNO $u^{\prime} g^{\prime} r^{\prime} i^{\prime} z^{\prime}$ data. After applying the transformations, there is a significant improvement in the average colors of the stellar locus of red stars. As shown in Figure 4, the PT data from Figure 1 align with the SDSS stellar locus when the new transformations are employed. Although the corrections from equations (3)-(6) are small, the offsets amount to more than $0.1 \mathrm{mag}$ in $(g-r)$ for the reddest stars. Corrections in the $(r-i),(i-z)$ diagram (Fig. 5) are less substantial, but still significant.

\section{DISCUSSION}

We have matched a large sample of background red stars observed with the PT to photometric observations of the same stars 

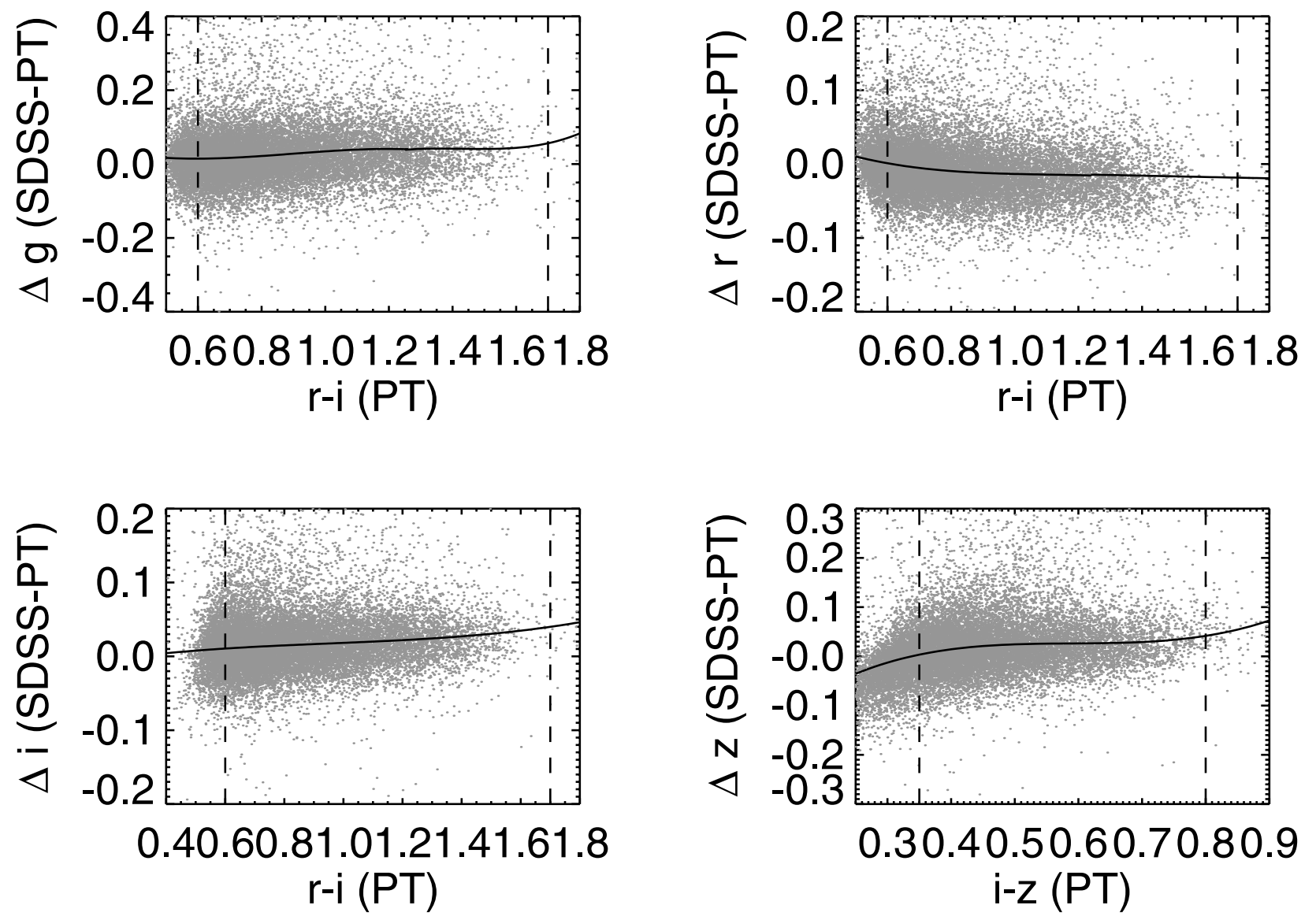

FIG. 3.- Difference in PT and SDSS magnitudes as a function of $(r-i)_{\mathrm{PT}}$ and $(i-z)_{\mathrm{PT}}$ color. Error-weighted polynomial least-squares fits are shown as solid lines in each panel. The vertical dashed lines indicate the color ranges over which the fits are reliable.

in the SDSS DR5 catalog. We present transformations based on magnitude differences between the $(\text { griz })_{\mathrm{PT}}$ and (griz) $)_{\text {SDSS }}$ photometric systems as functions of color.

These transformations provide a significant improvement in the photometric calibration of PT data onto the SDSS $2.5 \mathrm{~m}$ system for red stars. Our results augment the initial transformations described in T06, which have already been applied to all publicly available PT data. The systematic color offsets for red stars, cor-

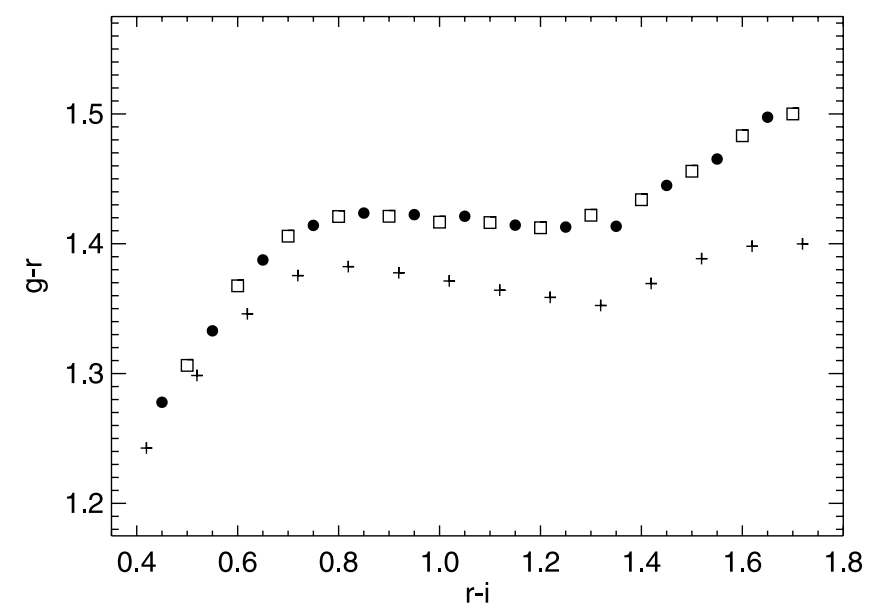

FIG. 4.- Median $(g-r),(r-i)$ colors of low-mass stars as in Fig. 1. The $g r i$ SDSS data are shown as open squares. The $g r i_{\mathrm{PT}}$ data prior to our corrections are shown as crosses, while gri $_{\mathrm{PT}}$ data transformed using the equations in $\S 3.2$ are shown as filled circles. responding to spectral types M0 through M5 (Bochanski et al. 2007a), which have previously been seen between the PT and SDSS (see Figs. 4 and 5), are corrected when our transformations are applied.

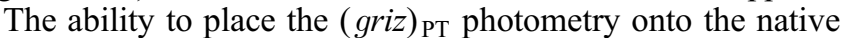
SDSS $2.5 \mathrm{~m}$ system allows PT observations to serve as a "bright extension" of the SDSS survey. In particular, PT observations of red stars with measured trigonometric parallaxes can now be reliably transformed onto the SDSS $2.5 \mathrm{~m}$ system with a typical accuracy $<10 \%$, allowing an improved definition of the color-magnitude

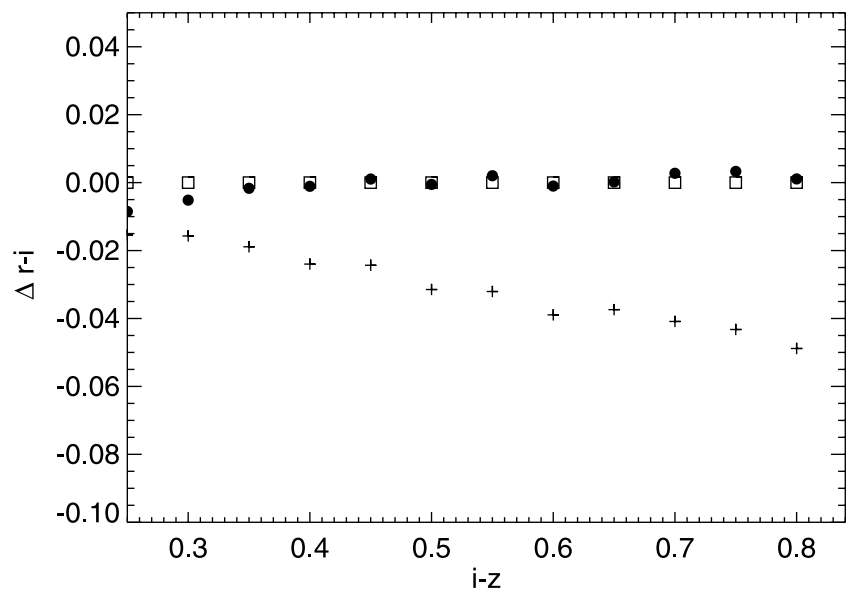

FIG. 5.- Median $\Delta(r-i),(i-z)$ residuals of low-mass stars. SDSS-SDSS data are shown as open squares. SDSS-PT data on the SDSS system prior to our corrections are shown as crosses, while SDSS-PT data transformed using the equations in $\S 3.2$ are shown as filled circles. 
relation for low-mass stars in native SDSS color space (Hawley et al. 2002; West et al. 2005; Davenport et al. 2006). We expect these results to be useful in analyzing targeted PT observations of red dwarf parallax standards (Williams et al. 2002; D. A. Golimowski et al. 2008, in preparation), as well as serendipitous PT observations of stars with Hipparcos parallaxes.

The authors would like to thank Douglas Tucker and David Golimowski for their insightful comments, suggestions, and assistance with MTPIPE. The authors acknowledge the support of NSF grants AST 02-05875 and AST 06-07644, and NASA ADP grant NAG5-1211. K. R. C. acknowledges that support for this work was provided by NASA through the Spitzer Space Telescope Fellowship Program, through a contract issued by the Jet Propulsion Laboratory, California Institute of Technology, under a contract with NASA. A. A. W. acknowledges the support of NSF grant AST 05-40567.

This project made extensive use of SDSS data. Funding for the SDSS and SDSS-II has been provided by the Alfred P. Sloan Foundation, the Participating Institutions, the National Science
Foundation, the US Department of Energy, the National Aeronautics and Space Administration, the Japanese Monbukagakusho, the Max Planck Society, and the Higher Education Funding Council for England. The SDSS Web site is at http://www.sdss.org/.

The SDSS is managed by the Astrophysical Research Consortium for the Participating Institutions. The Participating Institutions are the American Museum of Natural History, Astrophysical Institute Potsdam, the University of Basel, Cambridge University, Case Western Reserve University, the University of Chicago, Drexel University, Fermilab, the Institute for Advanced Study, the Japan Participation Group, Johns Hopkins University, the Joint Institute for Nuclear Astrophysics, the Kavli Institute for Particle Astrophysics and Cosmology, the Korean Scientist Group, the Chinese Academy of Sciences (LAMOST), Los Alamos National Laboratory, the Max Planck Institute for Astronomy, the Max Planck Institute for Astrophysics, New Mexico State University, Ohio State University, the University of Pittsburgh, the University of Portsmouth, Princeton University, the US Naval Observatory, and the University of Washington.

We finally would like to thank the anonymous referee for helpful comments and questions.

\section{REFERENCES}

Adelman-McCarthy, J., et al. 2007, ApJS, submitted

Bochanski, J. J., West, A. A., Hawley, S. L., \& Covey, K. R. 2007a, AJ, 133, 531

Bochanski, J. J., et al. 2007b, AJ, submitted

Chiu, K., Fan, X., Leggett, S. K., Golimowski, D. A., Zheng, W., Geballe, T. R., Schneider, D. P., \& Brinkmann, J. 2006, AJ, 131, 2722

Covey, K. C., et al. 2007, AJ, 134, 2398

Davenport, J. R. A., West, A. A., Matthiesen, C. K., Schmieding, M., \& Kobelski, A. 2006, PASP, 118, 1679

Fan, X. 1999, AJ, 117, 2528

Fukugita, M., Ichikawa, T., Gunn, J. E., Doi, M., Shimasaku, K., \& Schneider, D. P. 1996, AJ, 111, 1748

Gunn, J. E., et al. 1998, AJ, 116, 3040 2006, AJ, 131, 2332

Hawley, S. L., et al. 2002, AJ, 123, 3409

Hogg, D. W., Finkbeiner, D. P., Schlegel, D. J., \& Gunn, J. E. 2001, AJ, 122, 2129

Ivezić, Ž. et al. 2004, Astron. Nachr., 325, 583
Pier, J. R., Munn, J. A., Hindsley, R. B., Hennessy, G. S., Kent, S. M., Lupton, R. H., \& Ivezić, Ž. 2003, AJ, 125, 1559

Skrutskie, M. F., et al. 1997, in The Impact of Large Scale Near-IR Sky Surveys, ed. F. Garzon et al. (Dordrecht: Kluwer), 25

Smith, J. A., et al. 2002, AJ, 123, 2121

Stoughton, C., et al. 2002, AJ, 123, 485

Strauss, M. A., et al. 1999, ApJ, 522, L61

Tucker, D. L., et al. 2006, Astron. Nachr., 327, 821 (T06)

Walkowicz, L. M., Hawley, S. L., \& West, A. A. 2004, PASP, 116, 1105

West, A. A., Bochanski, J. J., Hawley, S. L., Cruz, K. L., Covey, K. R., Silvestri, N. M., Reid, I. N., \& Liebert, J. 2006, AJ, 132, 2507

West, A. A., Garcia-Appadoo, D. A., Dalcanton, J. J., Disney, M. J., Rockosi, C. M., Ivezic, Z., Bentz, C. M., \& Brinkmann, J. 2007, AJ, submitted

West, A. A., Walkowicz, L. M., \& Hawley, S. L. 2005, PASP, 117, 706

West, A. A., et al. 2004, AJ, 128, 426

Williams, C. C., et al. 2002, BAAS, 34, 1292

York, D. G., et al. 2000, AJ, 120, 1579 